\title{
In memoria di Gianfranco Folena
}

\author{
Pietro Folena
}

PUBBLICATO: 31 MAGGIO 2021

$\mathrm{D}$ evo anzitutto ringraziare l'Accademia della Crusca, e in particolare il Presidente Claudio Marazzini, per aver deciso di dedicare questa Tornata accademica alla memoria di Gianfranco Folena, e lo faccio anche a nome delle mie sorelle Lucia e Nora che ci stanno seguendo. Marazzini ha accettato l'anno scorso la proposta di guidare il Comitato per le celebrazioni del centenario della nascita di nostro padre, e di queste celebrazioni l'evento odierno è uno degli eventi più significativi. Dopo il grande convegno di tre giorni a Padova, nello scorso ottobre - grazie al lavoro prezioso del Circolo Filologico Linguistico, creatura prediletta di Gianfranco Folena - i contributi offerti oggi arricchiscono in modo assai significativo il campo della riflessione e della ricerca.

Desidero fare un grande plauso a maestri come Gianluigi Beccaria, Vittorio Coletti e Massimo Fanfani che hanno proposto delle relazioni di grande interesse, da studiare e da diffondere. Le testimonianze rese, poco fa, da Piero Fiorelli - di grande intensità emotiva -, da Lino Leonardi e da Francesco Sabatini hanno offerto spunti assolutamente originali, veri e propri impegni di lavoro e di approfondimento.

Questa Tornata coincide con la pubblicazione proprio oggi della nuova edizione, riveduta e corretta, de L'italiano in Europa. Da anni questo volume, che vinse il Premio Viareggio nel I983, era diventato introvabile. Voglio ringraziare l'editore Franco Cesati che con determinazione ha perseguito questo obiettivo e Daniela Goldin per il lavoro critico e di aggiornamento prezioso che è stato compiuto per realizzarlo.

Il nostro Comitato, come ha detto già Marazzini, ha un vasto programma di iniziative e di pubblicazioni per il 202I e per il 2022, a partire dal Convegno dell'Accademia dei Lincei annunciato per il prossimo mese di giugno.

Vorrei soffermarmi, in questo intervento, su un tratto di nostro padre che a me sembra determinante per spiegare come si sia potuto formare uno dei più grandi umanisti del Secondo Novecento. Beccaria e Coletti hanno parlato dei tratti paterni e fraterni che Gianfranco Folena aveva nello svolgimento del suo lavoro di ricerca e di insegnamento. Sono d'accordo. Tratti paterni e fraterni che ha avuto non solo nella sfera degli affetti - suo fratello, i suoi figli - ma anche, e direi soprattutto nella sfera culturale, che per lui è stata una dimensione morale, emotiva, sentimentale. Ha costruito rete, è stato detto, ben prima che nascesse il web: rete fra saperi e conoscenze, in un modo, spesso inconsapevole, straordinariamente attuale e innovativo.

Credo che occorra scavare nella sua biografia per comprendere meglio questo tratto. Nella sua biografia si sente la forza morale di quella generazione novecentesca che si è forgiata fra i due conflitti mondiali, durante la dittatura e la guerra, in esperienze di privazione materiale e degli affetti molto dure. Una generazione che ha ricostruito l'Italia, non solo dal punto di vista della sua dignità nazionale o da quello economico e sociale, ma anche e, per me, prima di tutto, secondo la lezione di Antonio Gramsci, dal punto di vista culturale. 
Il tratto prevalente della formazione del ragazzo e del giovane Folena è stato quello della solitudine. Fin da bambino l'aveva sperimentata.

Nel momento in cui l'Italia e il mondo erano in subbuglio, a casa Folena-Alberti, in Toscana, si soffriva per ragioni più intime.

Era durata poco la felicità per la sua nascita. Nora, la giovane e bellissima mamma, che nel ig22 aveva dato alla luce il fratello Alberto, mori di setticemia qualche settimana dopo. Il padre Umberto, medico militare, distrutto dal dolore, era sempre lontano.

I due piccoli maschi - Gianfranco e Alberto - si trovano così senza madre, e con un padre che, per ragioni di servizio e di lavoro, doveva ubbidire agli ordini della gerarchia. La nonna Giuseppina, madre di Nora, diventa per Gianfranco il simbolo della vita, e si creerà negli anni un legame intensissimo, testimoniato da una fitta corrispondenza.

Solo nelle lettere e nelle cartoline alla nonna si firmerà sempre Gianco. Solo per lei era Gianco. Gianfranco passa lunghi periodi con la famiglia della nonna, lontano dal fratello, che stava con altri zii. Gianfranco cresce in una grande solitudine, in un mondo dominato dalla nonna, e dalle donne, col padre assente, dimostrando capacità intellettuali fuori dal comune. Viene spinto dalla zia, che insegna al liceo a Grosseto, agli studi classici. La separazione dal fratello, verso il quale ha un istinto protettivo, come si percepisce dalla sua corrispondenza, è un dolore nel dolore. Si ritroveranno a vivere nella stessa città, molti decenni dopo, quando Alberto, che aveva compiuto studi nel settore, dopo aver pensato di ripercorrere le orme paterne, diventerà dirigente di una casa farmaceutica, e Gianfranco approderà invece nell'Ateneo patavino.

Ma è in tutta la sua crescita l'assenza della madre, combinata alla lontananza del padre, a determinare in modo indelebile la sua dedizione intellettuale, e più avanti a dare corpo ed anima al sogno di avere quella famiglia che lui non aveva conosciuto. "Colgo un momento in cui sono solo in casa - scrive da Cucigliana nel settembre del I934, quando aveva quattordici anni - per scriverti due righe tutte piene di nostalgia e di rimpianto di te, cara nonna, che un decennio fa mi raccogliesti privo della persona più cara e più buona": in quest'ultimo passaggio c'e tutto il tormento di un bambino diventato troppo presto grande, desideroso di concludere il liceo e di studiare all'Università. Lo studio diventa la ragione di vita, la missione laica di Gianfranco.

Mi piace qui ricordare l'amicizia profonda, negli anni del liceo, con Geno Pampaloni; e ancora il suo ingresso alla Scuola Normale, dove conoscerà Alessandro Natta, che era di due anni più grande. Quando Natta diventerà segretario del PCI - e io segretario della FGCI -, si riannoderà un amichevole rapporto epistolare tra di loro.

Ma i rumori, prima, e i drammatici eventi della guerra, poi, impediscono a quelle generazioni di compiere un normale corso di studi, di costruire un proprio progetto di vita. Tutto cambia in pochi mesi. Nostro padre va in guerra. In Libia viene fatto prigioniero dagli inglesi.

Già nel gennaio del 1943 si trova in India, prima al nord, in un campo nell'Uttarakhand, e l'ultimo periodo vicino a Bombay. Scrive a Geno Pampaloni, ("data la mancanza di libri e la sete ardente di lettura, ti prego di aiutarmi consigliando ai miei acquisti e invii...") chiedendogli di consigliare alla nonna i libri da spedirgli. Dall'Italia gli arrivano libri, cosi come ad altri compagni di prigionia, con cui si crea un legame speciale, da Ludovico Quaroni a Umberto Serafini. La chiamerà l'Università della prigionia. Qui si accentua, in condizioni obbligate, la sua missione culturale. Per tre anni, fino alla primavera del I946, ha l'opportunità di leggere e studiare i più svariati argomenti, e di confrontarsi 
con giovani con altre pulsioni intellettuali e vocazioni specialistiche. Ma in una dimensione di grande solitudine ("per lo più sto solo, se soli ci si può chiamare in una baracca di quaranta persone con uno spazio appena per il letto e per il tavolinetto", scrive al padre). La morte della madre e le privazioni negli affetti, e poi la lunga prigionia - cosi com’e stato per altri intellettuali rinchiusi in carcere - sono state le levatrici di questa personalità eccezionale.

Le sue lettere dalla prigionia meriteranno un esame approfondito. Sono venuto in possesso di alcune di esse solo in tempi recenti.

La prova fisica della prigionia è comunque molto dura, così come quella morale. Finalmente nell'aprile del I946, poco dopo il suo ventiseiesimo compleanno, si imbarca. Durante il viaggio gli giunge notizia della tragica morte del padre, investito in bicicletta da un carro armato americano. Il 23 aprile, sbarcato a Napoli, telegrafa alla nonna "saputo povero babbo", e poi "coraggio" rivolto a Lei.

Con fatica, e assorbendo questi nuovi dolori familiari e collettivi, Gianfranco riprende subito la sua strada. La formazione giovanile e l'Università della prigionia gli permettono rapidamente di concludere gli studi coi suoi grandi maestri, a partire da Bruno Migliorini. Nel dicembre di quello stesso terribile anno è già laureato. Qualche tempo dopo può telegrafare alla nonna con orgoglio per il suo nuovo incarico, dopo Grosseto, in un liceo di Lucca. Presto rientra a Firenze, comandato alla Crusca, dove compie in alcuni anni fondamentali ricerche e studi preziosi, e qui comincia una storia intellettuale più nota.

Ho avuto occasione, nel Convegno padovano, di ricordare cosa fu per lui l'incontro con nostra madre, intellettuale francese, e il cenacolo estivo che sulle rive della Loira si era venuto formando. Cosi come in quella occasione ho ricordato la sua passione politica e civile. Qui mi preme invece, per concludere, parlare di quella religione delle lettere e della cultura che ha occupato, talvolta interamente, la sua sfera emotiva, riempiendo di senso le perdite e la solitudine.

Mi è capitata fra le mani una lettera scritta a Lidia Migliorini in occasione della scomparsa di suo marito, Bruno, nel I975. Nostro padre si trova a Oxford, nel giugno del I975, dove insegna all'All Souls College. Non si può muovere in tempo per i funerali del suo Maestro. "Non mi vergogno di dirLe che da due ore piango come mi era successo solo due volte nella vita, per mio Padre, quando tornai dalla prigionia, e per Giorgio Pasquali: un destino ha voluto che sempre io fossi lontano in questi momenti e che dovessi consumare tutto da me il mio dolore".

Ecco: i tre padri di Gianfranco Folena. Quello biologico, Pasquali e Migliorini. E i molti figli - non solo noi, biologici, che abbiamo avuto la fortuna di averlo in casa -: gli allievi, le allieve, i nipoti, i folenotteri, e tutta una scuola che sorprendentemente, a quasi trent'anni dalla sua scomparsa, è ancora vitale.

Questo è successo perché nella forza della cultura, più che in quella degli affetti, dei quali era stato privato con durezza, ha trovato la ragione della sua vita.

Ricordando nel i972 un grande intellettuale scomparso qualche anno prima, Manlio Dazzi, comunista, scorgeva in quella figura non gia il dogmatico al servizio del partito, ma l'uomo che "sapeva che il simile si conosce non con il simile ma col diverso". Parlava di sé, in qualche modo, sostenendo che "col forte senso della socialità della cultura e delle lettere come bene comune e come servizio pubblico, il più alto di tutti i servizi" si ha la coscienza della vita come "contraddizione", nell'accezione dialettica e anche evangelica del termine. 
Questa religione delle lettere in mio padre prendeva la forma, come ha scritto qualche tempo fa Giulio Ferroni, di un amore sconfinato per la vita della parola, addirittura di profezia della lingua.

Questo amore nasceva in questa esistenza che, nelle sue vicende più dolorose, come in quelle più felici, l'aveva portato a considerare "la socialità della cultura come bene comune".

È stato supportato e sostenuto, in questo suo cammino. Ma ha trovato dentro di sé, nello studio, nell'insegnamento e nella ricerca una forza morale sorprendente.

\section{Cita come:}

Pietro Folena, In memoria di Gianfranco Folena, "Italiano digitale", XVII, 2021/2 (aprile-giugno) DOI: 10.35948/2532-9006/2021.7533

\section{Copyright 2021 Accademia della Crusca}

Pubblicato con licenza creative commons CC BY-NC-ND 